EPJ Web of Conferences 85, 02020 (2015)

DOI: $10.1051 /$ epjconf/ 20158502020

(C) Owned by the authors, published by EDP Sciences, 2015

\title{
Recent HERMES results from inclusive and semi-inclusive hadron production
}

\author{
Charlotte Van Hulse, on behalf of the Hermes Collaboration \\ University of the Basque Country - UPV/EHU, Spain \\ e-mail: cvhulse@mail.desy.de
}

\begin{abstract}
Hermes collected a wealth of data using the $27.6 \mathrm{GeV}$ polarized Hera lepton beam and various pure, polarized and unpolarized, gaseous targets. This unique data set opens the door to various measurements sensitive to the multi-dimensional structure of the nucleon. Among them are two-hadron production in semi-inclusive deep-inelastic scattering on a transversely polarized target, providing access to different transverse-momentumdependent distribution and fragmentation functions in a way complementary to single-hadron production. Also transverse-target single-spin asymmetries in inclusive electroproduction of charged pions and kaons have been recently extracted, complementing data from proton-proton collisions, where large, so far unexplained, asymmetries have been observed. From inclusive measurements on various nuclear targets, the $\Lambda$ polarization is extracted, also here complementing measurements in proton-proton collisions.
\end{abstract}

\section{Introduction}

The Hermes experiment at Desy in Hamburg (Germany) collected data from 1995 until 2007 using the $27.6 \mathrm{GeV}$ Hera lepton beam. In the experiment, longitudinally polarized electrons or positrons were scattered off stationary gaseous hydrogen, deuterium, helium, or heavier targets, with hydrogen longitudinally or transversely polarized or unpolarized, deuterium and helium longitudinally polarized or unpolarized, and the heavier targets unpolarized. The scattered lepton and particles produced in the collision were detected by a forward spectrometer. Here, leptonhadron separation was performed by a transition-radiation detector, a preshower, and a calorimeter, with an identification efficiency exceeding $98 \%$ and a misidentification contamination below $1 \%$. Hadron identification was performed by a ring-imaging Cherenkov detector, allowing the discrimination of pions, kaons, and protons.

From data collected on the transversely polarized hydrogen data, transverse-target moments of two hadrons were extracted. These provide access to distribution and fragmentation functions, in a way complementary to single-hadron production. Results on these moments for pions and kaons are presented in section 2 .

Motivated by the unexplained large left-right asymmetries observed in, e.g., proton-proton collisions, with one of the protons transversely polarized, azimuthal singlespin asymmetries in inclusive electroproduction of pions and kaons off transversely polarized protons were extracted. This is reported in section 3.

Finally, measurements of transverse $\Lambda$ polarization in quasi-real photoproduction on various nuclei were finalized, also here complementing measurements in protonproton collisions. This is presented in section 4 .

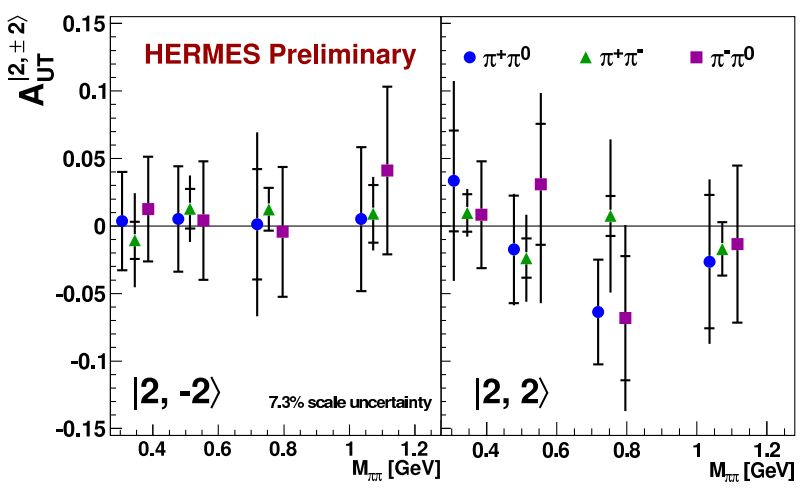

Figure 1. Collins $\mid 2, \pm 2>$ transverse-target moments for pion pairs.

\section{Two-hadron production in semi-inclusive deep-inelastic scattering}

Two-hadron production in semi-inclusive deep-inelastic scattering on a transversely polarized target allows to access the same distribution functions as in singlehadron production, while providing additional information. Through two-hadron production different flavor combinations can be accessed compared to single-hadron production. Moreover, in two-hadron production transversity enters the cross section colinearly, through a direct product, whereas for single-hadron production transversemomentum-dependent convolution integrals enter the cross section, thereby largely complicating access to transversity.

The underlying theoretical framework of the here presented analysis for the expression of the transversemomentum-dependent two-hadron semi-inclusive deep- 


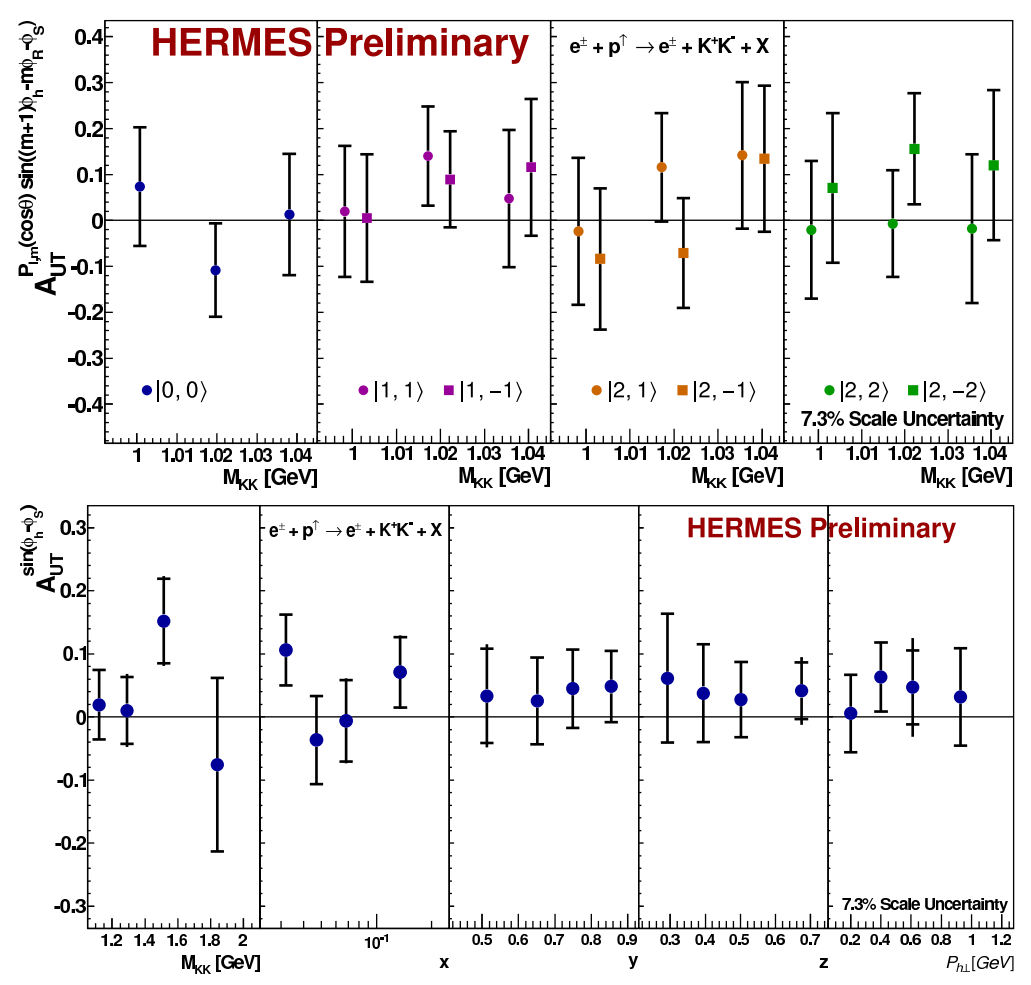

Figure 2. Sivers transverse-target moments for kaon pairs in the $\phi$-resonance region (top) and outside the $\phi$-resonance region (bottom).

inelastic scattering cross section is developed in Ref. [1]. Here, a slight modification of the definition of fragmentation functions, compared to e.g. Ref. [2], is introduced. At leading twist three classes of fragmentation functions appear: the unpolarized fragmentation function, which corresponds to the sum of diagrams without quark helicity flip, the polarized fragmentation function, which corresponds to the difference of these same diagrams, and finally the generalized Collins fragmentation function, which corresponds to the sum of diagrams with quark helicity flip. Each class of fragmentation functions is expanded in partial waves on the basis of spherical harmonics. As detailed in Ref. [1], this redefinition not only may help in the interpretation and presentation of cross-section moments, but it also allows to derive the two-hadron semiinclusive deep-inelastic scattering cross section from the single-hadron semi-inclusive deep-inelastic cross section, and this at any twist level.

Data collected on a transversely polarized hydrogen target with an electron and positron beam were used to extract di-hadron moments in semi-inclusive deep-inelastic scattering. The di-hadrons consist of pairs of charged and neutral pions in the combinations $\pi^{+} \pi^{-}, \pi^{+} \pi^{0}$, and $\pi^{-} \pi^{0}$, and of pairs of charged kaons, $K^{+} K^{-}$. The invariant-mass spectra of the pion pairs receives, besides the non-resonant contribution, various resonant contributions of unstable mesons, such as $\rho, \eta$ and $\omega$ mesons. For the kaon spectrum only the $\phi$ resonance and non-resonant contributions dominate. The $\phi$ resonance, being a nearly pure $s \bar{s}$ state, is of particular interest as it allows access to the Sivers and Collins functions for strange quarks.
The extraction of the moments is done in onedimensional binning in $M_{h h}$ and in two-dimensional binning in $M_{h h}$ and either of the variables $x, y, z$, or $P_{h \perp}$ for the pion pairs and for the kaon pairs near the $\phi$ resonance, i.e., with $M_{K K}<1.05 \mathrm{GeV}$. Here, $M_{h h}$ is the invariant mass of the considered hadron pair, $x$ is the $x$-Bjorken variable, $y$ represents in the target-rest frame the fractional virtual-photon energy with respect to the beam-lepton energy, $z$ denotes in the target-rest frame the fractional hadron-pair energy with respect to the virtualphoton energy, and $P_{h \perp}$ is the magnitude of the component of the sum of the hadron momenta that is transverse to the virtual-photon three-momentum in the plane spanned by the three-momentum of the virtual photon and the sum of the momenta of the hadron pair. For the kaon pairs outside the $\phi$ resonance region, i.e., for $M_{K K}>1.05 \mathrm{GeV}$, only a one-dimensional extraction in any of the above five described variables is performed. The presence of nonresonant contributions only and the lower amount of data available for kaons compared to pions motivates this approach.

Figure 1 shows the transverse-target moments $\mid 2, \pm 2>$ of the Collins function for each of the pion pairs. As can be seen, the moments corresponding to $(l=2, m=-2)$ are consistent with zero. For the ( $l=2, m=2)$ moments no signal outside the $\rho$-resonance region is observed, while there is a hint of a negative moment value for $\pi^{ \pm} \pi^{0}$ in the $\rho^{ \pm}$region. For the $\rho^{0}$ resonance region the moment is consistent with zero. Assuming dominance of scattering off a $u$ quark, the negative value for $\pi^{+} \pi^{0}$ in the $\rho^{+}$region can be explained through the Artru model [3], which predicts an 


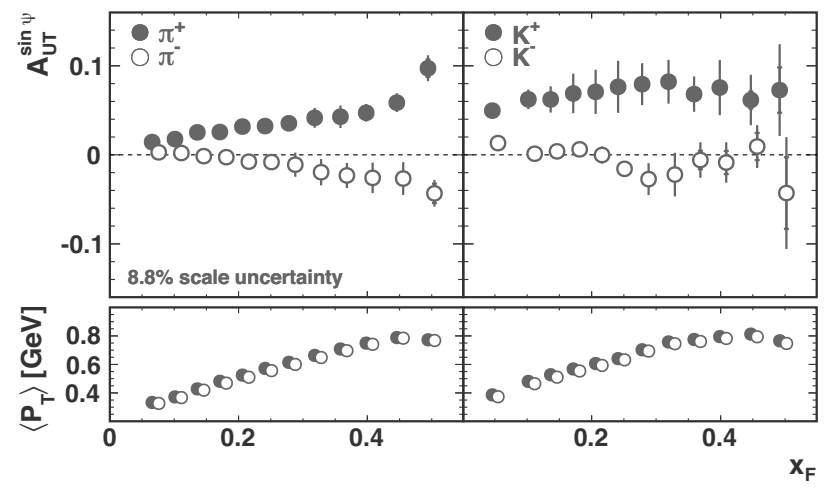

Figure 3. Transverse-target asymmetry amplitudes for charged pions and kaons in inclusive electroproduction as a function of $x_{F}$ (top). Average $P_{T}$ values as a function of $x_{F}$ (bottom).

opposite sign for favored production of vector mesons and of pseudo-scalar mesons. Under the hypothesis of $u$-quark scattering dominance and given the results for Collins moments for single-pion production [4], the behavior of $\pi^{-} \pi^{0}$ cannot be explained through the Artru model.

Figure 2, top, presents Sivers moments for kaon pairs in the $\phi$-resonance region. A signal consistent with zero is observed for all moments, which hints at a very small value of the product of the $s$-quark Sivers distribution and the unpolarized fragmentation function. An analogous behaviour is seen for the Collins moments (not shown here), hinting at a small transversity distribution in combination with the Collins fragmentation function for $s$ quarks. As can be seen from figure 2, bottom, outside the $\phi$-resonance region the $\mid 0,0>$ moments show a small positive value. The other moments have been verified to be consistent with zero, as expected. Also here, for the Collins moments an analogous behaviour is observed for the $\mid 0,0>$ moments as well as for the other moments.

\section{Transverse-target single-spin asymmetry in inclusive electroproduction of charged pions and kaons}

Various polarized proton-proton scattering experiments, with center-of-mass energies ranging from $5 \mathrm{GeV}$ to $200 \mathrm{GeV}$, have over the last 35 years observed large leftright asymmetries. These large observed values are not interpretable in leading-twist based on collinear factorization. Instead, approaches based on transverse-momentumdependent distribution and fragmentation functions [5] or on collinear parton dynamics linked to higher-twist multiparton correlations [6-11] could offer an explanation. At present, however, no conclusive statement about the correct interpretation can be made, and more experimental data are needed.

Complementing measurements made in hadronhadron collisions and hadron production in semi-inclusive deep-inelastic scattering, here single-spin asymmetries in inclusive electroproduction of charged pions and kaons off a transversely polarized proton target are presented. These asymmetries are extracted as a function of $P_{T}$, the transverse-momentum component of the hadron with respect to the beam direction, and $x_{F}$ the Feynman variable calculated in the lepton-nucleon center-of-momentum frame [12].

The asymmetries extracted as a function of $x_{F}$ for charged pions and kaons are presented in the upper panels of figure 3. As can be seen, for positive pions the amplitude is positive and increases linearly with increasing $x_{F}$, while for negative pions the amplitude is negative and decreases linearly with increasing $x_{F}$. The observed behaviour as a function of $x_{F}$ is similar to the behaviour observed in hadron-hadron collisions. For positive kaons a positive amplitude value, approximately constant with $x_{F}$, is seen, while negative kaons show an amplitude value compatible with zero, exhibiting small variations over $x_{F}$. In the lower panels the correlation between $P_{T}$ and $x_{F}$ is displayed. The strong correlation between these two variables illustrates the need for a two-dimensional extraction.

The two-dimensional extraction is presented in figure 4 , where the asymmetry amplitudes are shown as a function of $P_{T}$ for different slices of $x_{F}$. The amplitude for positive pions increases with $P_{T}$ up to a $P_{T}$ value of $0.8 \mathrm{GeV}$ and then decreases. At high values of $P_{T}$ there is an indication that the asymmetry amplitude rises again. The observed $P_{T}$ dependence is independent of $x_{F}$, thus clarifying that the observed dependence as a function of $x_{F}$ in the one-dimensional extraction solely stems from the underlying $P_{T}$ dependence. For negative pions small amplitudes, varyingly positive and negative with $P_{T}$, are observed. Here, however, a dependence on $x_{F}$ is observed, i.e., the amplitudes decrease with increasing $x_{F}$. As for positive pions, positive kaons display positive, albeit slightly larger, amplitude values increasing with $P_{T}$, up to $P_{T}=0.8 \mathrm{GeV}$, and then decreasing again. Contrary to positive pions, positive kaons exhibit a slight dependence on $x_{F}$, with amplitudes increasing with increasing values of $x_{F}$. For negative kaons, small amplitudes are observed, decreasing slightly with increasing $x_{F}$.

The extracted inclusive data set presumably contains a mixture of various contributing processes. In order to disentangle the different contributions, the existing data set is subdivided according to different requirements. One sample, the anti-tagged sample, contains events in which no electron or positron is detected. This corresponds predominantly to events with $Q^{2} \approx 0$, where $Q^{2}$ represents the negative squared four-momentum of the virtual photon that mediates the lepton-nucleon interaction. The subsequent samples consist of events for which the scattered lepton is detected and that satisfy the conditions of the deep-inelastic scattering regime. Here, for one sample it is required that $z$, which denotes in the target-rest frame the fractional hadron energy with respect to the virtualphoton energy, is comprised between 0.2 and 0.7 , while for the other category of events it is required that $z>0.7$. The asymmetry amplitudes for the various subsamples are shown in figure 5 for pions and kaons as a function of $P_{T}$ together with the fractions of the deep-inelastic scattering 


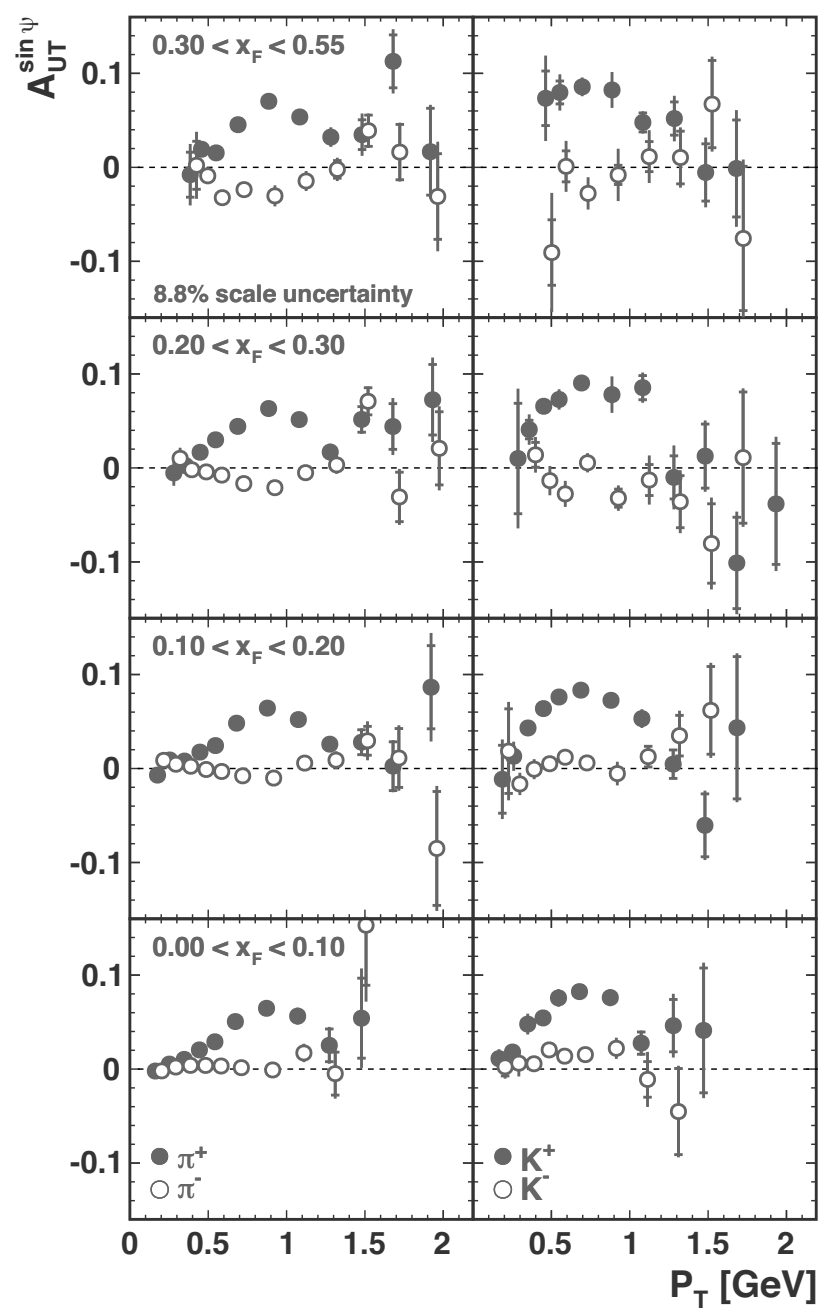

Figure 4. Transverse-target asymmetry amplitudes for charged pions and kaons in inclusive electroproduction as a function of $P_{T}$ for different slices in $x_{F}$.

samples with respect to the total inclusive sample. As can be seen, the fractional contributions of the deep-inelastic scattering samples increase with increasing $P_{T}$, and for $P_{T}$ values above $1.5 \mathrm{GeV}$, the sample with $z>0.7$ dominates over the sample with $0.2<z<0.7$ for positive hadrons. As for the asymmetry values, the results from the antitagged sample follow the overall inclusive results. Since the former comprises about $98 \%$ of the whole statistics, this behaviour is easily understood. For the deep-inelastic scattering data sample with $0.2<z<0.7$, results similar to the Sivers amplitudes [13] are observed. Since the azimuthal angles of the here extracted asymmetry and of the Sivers amplitude are closely related, this does not come unexpected. For the sample with $z>0.7$, large asymmetries are observed for both pions and especially for positive kaons. This subsample receives contributions from various processes. For pions, exclusive mesons can contribute, with decaying $\rho$ mesons contributing up to $30 \%$ and $50 \%$ for positive and negative pions, respectively. For kaons, the contribution from $\phi$ decays amounts to less than $10 \%$. Also, at this high values of $z$, the role of favoured fragmentation might play a dominant role.

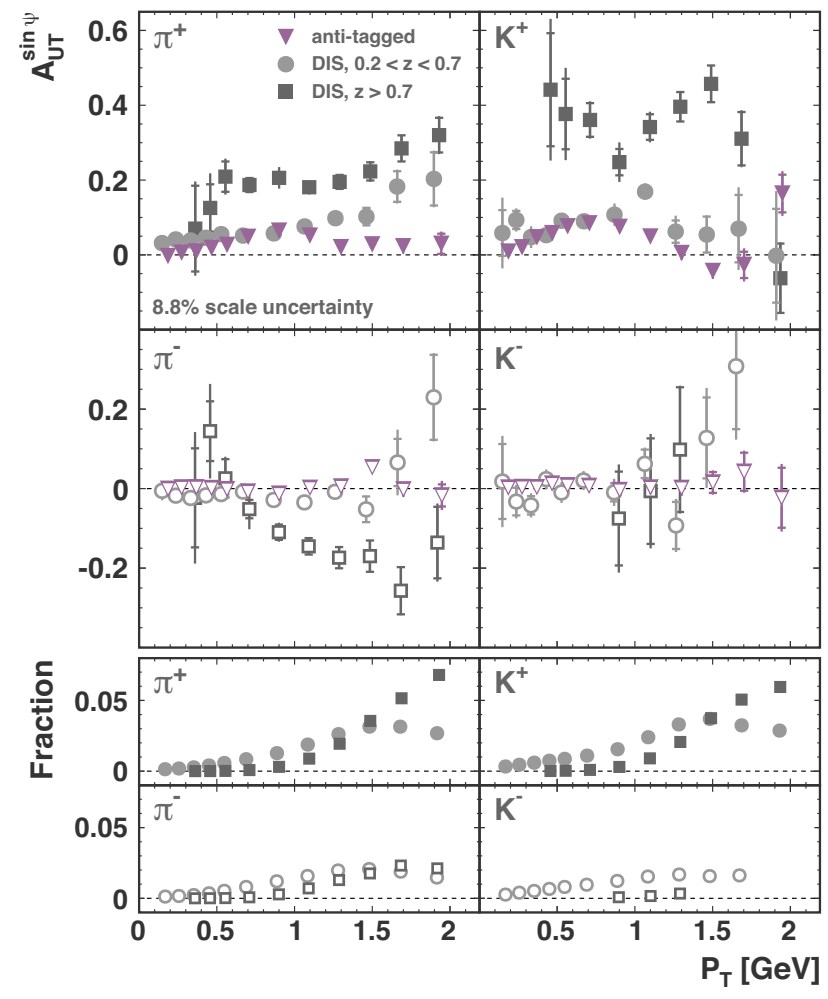

Figure 5. Transverse-target asymmetry amplitudes for charged pions and kaons in inclusive electroproduction as a function of $P_{T}$ for various subsamples, i.e., the anti-tagged sample and the deep-inelastic scattering samples with $0.2<z<0.7$ and $z>0.7$ (top). The fraction of the latter two samples with respect to the total inclusive sample (bottom).

\section{Transverse polarization of $\Lambda$ hyperons from quasi-real photoproduction on nuclei}

High transverse-polarization values, of up to $40 \%$, of $\Lambda$ hyperons produced in hadron-nucleon, hadron-nucleus and nucleus-nucleus collisions have been observed [1416]. While in hadron-hadron collisions the transverse polarization of $\Lambda$ hyperons has been extensively studied, transverse $\Lambda$ polarization in photo- and electroproduction is little known. In the present section, results on the transverse polarization of $\Lambda$ hyperons produced in inclusive quasi-real photoproduction on various targets, i.e., hydrogen, deuterium, helium, neon, krypton, and xenon are discussed [17].

Figure 6 presents the measured $\Lambda$ polarization values as a function of the atomic-mass number $A$ for the various target nuclei hydrogen, deuterium, helium, neon, krypton, and xenon. Positive polarization values are observed for light nuclei, while they are consistent with zero for the heavier targets krypton and xenon. There is, despite the rather large polarization for neon, an indication of decreasing polarization with increasing atomic-mass number.

In figure 7 the polarization values for combined hydrogen and deuterium data and combined krypton and xenon data are shown as a function of $p_{T}$, where $p_{T}$ represents the $\Lambda$ momentum component transverse to the beam di- 


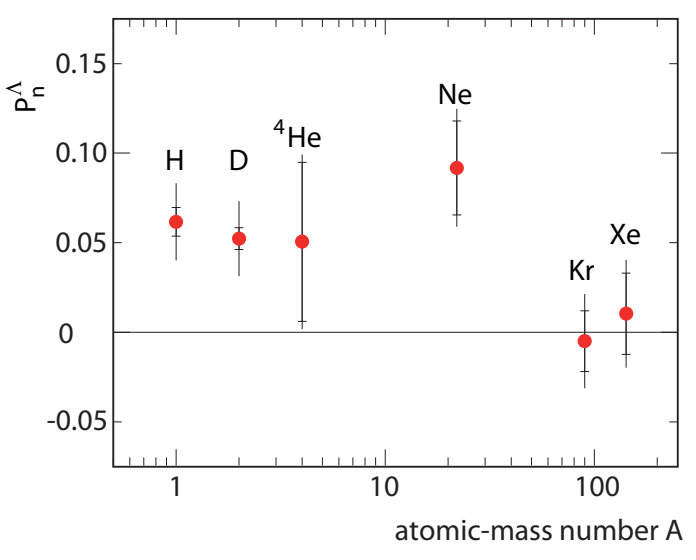

Figure 6. Transverse polarization of $\Lambda$ hyperons as a function of the atomic-mass number $A$.

rection. The results for hydrogen and deuterium are shown for different ranges of $\zeta$, with $\zeta=\left(E^{\Lambda}+p_{L}\right) /(E+k), E^{\Lambda}$ the energy of the produced $\Lambda$ hyperon, $E$ and $k$ the energy and momentum of the beam lepton, and $p_{L}$ the $\Lambda$ momentum component parallel to the beam direction as measured in the target rest frame. For values of $\zeta$ above 0.3 , the $\Lambda$ hyperon is predominantly produced in the forward region in the virtual-photon-nucleon center-of mass frame, while for $\zeta<0.2$ it is predominantly produced in the backward region. As can be seen, the $\Lambda$ polarization extracted from hydrogen and deuterium data shows a different behaviour in the forward and backward region as a function of $p_{T}$. For the backward region a clear, linear, rise of the polarization as a function of $p_{T}$ is observed, up to a polarization value of 0.12 for $p_{T}=0.75 \mathrm{GeV}$. In the forward region the polarization value is substantially smaller and no clear dependence on $p_{T}$ can be distinguished. For the krypton and xenon data, the statistical uncertainties prevent any conclu-

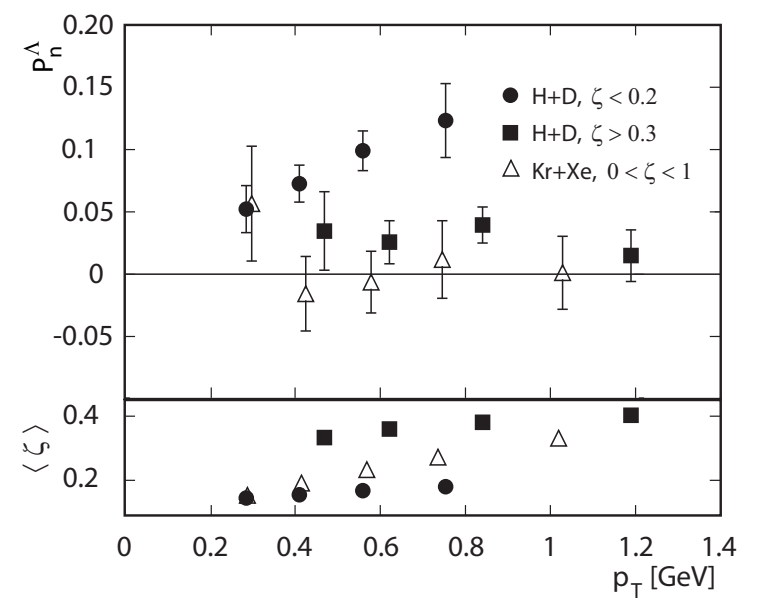

Figure 7. Transverse polarization of $\Lambda$ hyperons as a function of their transverse momentum for hydrogen and deuterium data in two different $\zeta$ regions and for krypton and xenon data. sion with respect to the $p_{T}$ dependence in the two regions of $\zeta$. In the entire $\zeta$ region, the polarization is seen to be independent of $p_{T}$ and compatible with zero.

\section{References}

[1] S. Gliske, A. Bacchetta, and M. Radici, arXiv:1408.5721 (2014).

[2] A. Bacchetta and M. Radici, Phys. Rev. D69, 074026 (2004).

[3] X. Artru, J. Czyzewski, and H. Yabuki, Z. Phys. C73, 527-534 (1997).

[4] A. Airapetian et al., Phys. Lett. B 693, 11-16 (2010).

[5] M. Anselmino, M. Boglione, and F. Murgia, Phys. Lett. B 362, 164-172 (1995).

[6] J. Qiu and G. F. Sterman, Phys. Rev. D59, 014004 (1999).

[7] Y. Kanazawa and Y. Koike, Phys. Lett. B 478, 121-126 (2000).

[8] Y. Kanazawa and Y. Koike, Phys. Lett. B 490, 99-105 (2000).

[9] Y. Koike, Nucl. Phys. A 721, 364-367 (2003).

[10] Y. Koike, AIP Conf. Proc. 675, 449-453 (2003).

[11] C. Kouvaris et al., Phys. Rev. D74, 114013 (2006).

[12] A. Airapetian et al., Phys. Lett. B 728, 183-190 (2014).

[13] A. Airapetian et al., Phys. Rev. Lett. 103, 152002 (2009).

[14] L. Pondrom, Phys. Rep. 122, 57 (1985).

[15] A. D. Panagiotou, Int. J. Mod. Phys. A 5, 1197 (1990).

[16] V. V. Abramov, Physics of Atomic Nuclei 72, 1872 (2009).

[17] A. Airapetian et al., arXiv:1406.3236. 